\title{
Investigating crystal microstructure of niobium materials by an $x$-ray diffraction reciprocal space mapping technique
}

\author{
Qiguang Yang, ${ }^{1}$ Frances Williams, ${ }^{1}$ Xin Zhao, ${ }^{2, *}$ Charles Reece, ${ }^{2}$ and Mahadevan Krishnan ${ }^{3}$ \\ ${ }^{1}$ Center for Materials Research, Norfolk State University, Norfolk, Virginia 23504, USA \\ ${ }^{2}$ Thomas Jefferson National Accelerator Facility, Newport News, Virginia 23606, USA \\ ${ }^{3}$ Alameda Applied Sciences Corporation (AASC), San Leandro, California 94577, USA
}

(Received 20 August 2013; published 10 January 2014)

\begin{abstract}
An x-ray diffraction reciprocal spacing mapping (RSM) technique was applied to investigate crystal quality of $\mathrm{Nb}$ films and bulk materials, relevant to superconducting radio frequency applications. The RSM study used different x-ray penetration depths to study material processes after two different surface treatments: mechanical polishing or energetic condensation (a plasma process). The study revealed the material's microstructure evolution for the two different processes. The RSM plots revealed crystal quality of the materials at different thickness. The novel, differential-depth RSM technique presented in this study substantiated the following facts: (1) For a heteroepitaxial $\mathrm{Nb}$ film $\mathrm{Nb}(100) / \mathrm{MgO}(100)$, a film thickening process, via a cathodic arc-discharge $\mathrm{Nb}$ ion deposition (energetic condensation), created a near-perfect single crystal $\mathrm{Nb}$ on the surface top layer. (2) For a fine mechanically polished single-crystal bulk $\mathrm{Nb}$ material, the microstructure on the top surface layer is more disordered than that at a greater depth.
\end{abstract}

DOI: 10.1103/PhysRevSTAB.17.013501

PACS numbers: $81.15 . \mathrm{Fg}, 68.55 . \mathrm{J}-$

\section{INTRODUCTION}

Superconducting radio frequency (SRF) accelerating cavities for particle accelerators that are made of a bulk niobium $(\mathrm{Nb})$ material or with a $\mathrm{Nb}$ thin film coated on $\mathrm{Cu}$ or other substrates, are used in state-of-the-art facilities for exploring frontier physics, such as the Continuous Electron-Beam Accelerator Facility at the Thomas Jefferson National Accelerator Facility (Jefferson Lab), the European X-ray Free Electron Laser, the Facility for Rare Isotope Beams, the Spallation Neutron Source, and the proposed International Linear Collider. $\mathrm{Nb}$ film coated copper cavities had earlier successfully served the Large Electron Positron Collider project at CERN in the 1990s.

Next-generation SRF particle accelerators call for revolutionary new materials or new surface treatment processes, in order to be more energy efficient and be more materials-cost frugal.

To design and develop an optimal SRF material process, it is necessary to adopt new analytic techniques to nondestructively characterize the SRF functional layer less than $200 \mathrm{~nm}$ into the surface. One is interested in understanding the crystal quality of the superconductor within this depth.

The definition and measurement of crystal quality in this text is based on crystallographic long-range order (coherence) of atoms, but not on chemical impurities. As was pointed out by Cullity et al. [1,2], the quality of so-called

\footnotetext{
*xinzhao@jlab.org

Published by the American Physical Society under the terms of the Creative Commons Attribution 3.0 License. Further distribution of this work must maintain attribution to the author(s) and the published articles title, journal citation, and DOI.
}

"single crystals" or grains, varies from one case to another. At one extreme, a grain might have gone through plastic deformation, such that some portions (subgrains) of the grain are misoriented from the others, thus the dislocation density is high; at the other extreme, some precisely grown crystals have ultralow density of dislocations, line or planar imperfections (stacking faults), and their crystal planes in that single grain are flat to less than $10^{-4 \circ}$ over a distance of centimeters. Essentially, a motivation of this study is to quantify the density of intragrain defects in $\mathrm{Nb}$ materials by diffraction techniques.

In recent years, our research found that energetic condensation is a practical way to create high quality $\mathrm{Nb}$ film materials [3-7]. Energetic condensation has been accomplished by a cathodic-arc-discharge [7] or an electron cyclotron resonant $\mathrm{Nb}$ plasma ion source [8]. To understand the structural evolution of SRF $\mathrm{Nb}$ films grown via energetic condensation is an important and challenging task.

To characterize SRF materials, one convenient choice in the SRF community is the electron backscattering diffraction (EBSD) technique. EBSD has been widely used [9] to investigate the microstructure of $\mathrm{Nb}$ materials (thin film or bulk). The probing depth of EBSD is $\sim 50 \mathrm{~nm}$, which is comparable to the SRF London penetration depth. Therefore, EBSD measurements have provided useful insights into SRF materials in many studies $[10,11]$.

A popular choice of the EBSD tool, is commercial software of the EDAX/TSL company. The program is called OIM Data Collections and Analysis. The OIM stands for crystallographic "orientation indexing mapping" of crystals, which is the core value of a commercially available EBSD toolbox. It is to be noted that a plurality of grains are surveyed in a conventional EBSD study. 
The limit of the EBSD technique is that the state-of-art EBSD instrument is mainly applied to visualize orientation of multiple crystals or grains (intergrains' characteristics, grain boundaries angles, grain size etc.). A single crystal's quality, or intragrain's density of structural defects is beyond the primary scope of the OIM technique. Only a few indirect parameters in the EBSD OIM software, such as crystal-orientation indexing confidence index (CI) or Kikuchi pattern imaging quality (IQ), extracted from the EBSD imaging data set, might shed qualitative light on intragrain crystal quality. To relate our measurements to conventional EBSD mapping techniques, we have presented the measured CI and IQ values as benchmarks. Thus, the EBSD technique has its limitation as a tool for characterizing the individual grains or crystals.

Research on crystal quality is sensitive to subtle deviation of crystal planes (e.g., bending, twisting, polygonization) or plane distance ( $d_{h k l}$, which is proportional to the lattice constant). Outside the SRF community, in scientific research of semiconductors [12-16], macromolecular crystal proteins [2], and optic electronics [17], the x-ray diffraction reciprocal space mapping technique (XRD RSM) has been widely utilized, for example, to measure the structural properties of epitaxial films, or to reveal film composition, layer tilting, lattice relaxation, and crystal quality. Such RSM plots could demonstrate the strain, relaxation, and misorientation of a thin film on a substrate. The detection area of an x-ray machine could be a few square centimeters. In a RSM plot, both $\Delta \theta_{h k l}$ (lattice constant deviation) and $\Delta \omega$ (crystal planes misorientation) are observable. Different structural properties may be revealed in one graph.

Here we describe a novel RSM technique, using x-ray penetration of a sample (film or bulk) to different depths. The depth variation is accomplished by varying the incident angle of the x-rays. Thus this new technique allows nondestructive investigation of a material's structural character at different depths. It can reveal structural evolution of a SRF material after different processes, such as film coating, polishing, or heating.

\section{EXPERIMENTAL METHOD}

To validate the use of RSM for Nb materials study, three types of representative coupon samples were selected. They went through different materials processes. It is expected that these representatives have distinct microstructure character in surface and in the bulk layer. For this study, the surface thickness of interest is less than $100 \mathrm{~nm}$, because only this depth is relevant to the RF London penetration depth in a SRF application.

Film samples dubbed CED-34, CED-38, and CED-47 are $\mathrm{Nb}$ films that were coated on single crystal $\mathrm{MgO}$ (100) substrates using energetic condensation [5]. The bulk sample $\mathrm{Nb}-\mathrm{SC}-01$ is a single crystal $\mathrm{Nb}$ coupon. Sample Nb-PC-01 is a polycrystalline bulk Nb coupon.
The deposition method of making film samples has been described elsewhere $[5,18]$. The energetic condensation was conducted via a cathodic arc-discharge $\mathrm{Nb}$ ion deposition. Such a $\mathrm{Nb}$ plasma ion uses a low voltage (30 V) arc discharge to generate highly ionized plasma. The energy spectrum of ions from coaxial energetic deposition (CED) ranges from $20 \mathrm{eV}$ up to $170 \mathrm{eV}$. The $\mathrm{CED}$ facility was run in a pulse mode, and the pulse lasts $1 \mathrm{~ms}$ during which $\sim 0.5 \mathrm{~nm}$ of $\mathrm{Nb}$ is deposited on the substrate. The instantaneous deposition rate is as high as $560 \mathrm{~nm} / \mathrm{s}$. The estimated (instantaneous) ion flux is $10^{18} \mathrm{Nb}$ ions $/ \mathrm{cm}^{2}-\mathrm{s}$. The repetition rate (frequency) of the arc or pulse is $0.5 \mathrm{~Hz}$, limited by silicon controlled rectifier switches, but may be increased by using insulated gate bipolar transistor switches to more rapidly turn off the arc between pulses. By considering the dead time and frequency of the arcs, the nominal deposition rate is $\sim 3 \AA / s$, or roughly one atomic monolayer per second. This nominal deposition rate is similar to a conventional physical vapor deposition method, such as a magnetron sputtering source $(1.67 \mathrm{~nm} / \mathrm{s})$ or the electron cyclotron resonant $\mathrm{Nb}$ plasma source at Jefferson Lab $(2.25 \mathrm{~nm} / \mathrm{s})$.

The bulk $\mathrm{Nb}$ coupons Nb-SC-01 and Nb-PC-01 $(10 \times 10 \times 3 \mathrm{~mm})$ were chemically mechanically polished (CMP'ed process) at the WahChang company. The CMP is a "mirror-finishing" process to obtain an ultraflat surface. After CMP, the samples were lightly chemically etched for a few $\mu \mathrm{m}$ to remove the "damage layer" that is caused by the mechanical polish. The etch used was the typical "buffered chemical polish" (BCP) applied to niobium. After the BCP process, most of the impurity residuals or crystallographic defects were removed. The surface appeared very shiny and morphologically flat under the scanning electron microscope. The samples' surface roughness $\left(R_{\mathrm{RMS}}\right)$ measured by atomic force microscopy, are $\sim 40 \mathrm{~nm}$ on a $50 \times 50 \mu \mathrm{m}$ survey area.

The residual resistance radio (RRR) values of the $\mathrm{Nb}$ film samples were measured at the vertical cryogenic testing area of Jefferson Lab. The testing methods have been reported elsewhere $[5,18]$. As a measurement of direct current electrical properties, this value embodies the density of structural defects of a conductive sample (usually a metal). In this study, the RRR value is defined as the ratio of resistivity at $300 \mathrm{~K}$ to that at $10 \mathrm{~K}$. Noticeably, because our measurement obtains a RRR value across the entire film thickness, about a few $\mu$ m, this bulk property (RRR) is different [5] from a local zone RRR (such as the surface layer's RRR). In this research, only the RRR of film samples (not bulk ones) were tested and reported.

Pole figure and reciprocal space mapping measurements were performed at Norfolk State University using a high resolution four-circle PANalytical X'Pert Pro Materials research diffracto meter. The system was especially designed for thin film analysis and operated with a $\mathrm{Cu}$ $K_{\alpha}$ X-ray source at a wavelength of $1.54 \AA$ Å.Unless specified 
otherwise, the $\mathrm{x}$-ray beam projection area on a sample was $2 \times 2 \mathrm{~mm}$. The incident optics was a high-resolution four bounce $\mathrm{Ge}(220)$ monochromator, and the diffraction optics was a high resolution triple axis/rocking curve element.

Figure 1 shows a standard XRD experimental arrangement. The angle $\theta_{h k l}$ stands for the diffraction angle of $\{h k l\}$ Bragg planes; $\omega$ is an x-ray beam incident angle; $\phi$ is an azimuth angle of the sample stage; $\psi$ is a tilting angle against the normal direction of the sample stage.

To investigate the FWHM of a single XRD peak, the scan step was set to $0.003^{\circ}$ to fully use the high resolution of the system. The scan step was changed to $0.01^{\circ}$ for a full range $\theta-2 \theta$ scan, $0.06^{\circ}$ for reciprocal space mapping measurement, $0.1^{\circ}$ for $\phi$ scan, and $2^{\circ}$ for pole figure measurements.

An XRD pole figure is particularly useful to measure the texture of polycrystalline materials (aka. preferential orientation), or misorientation of a single crystal film against a cut plane. The principles of pole figure surveys can be found in an x-ray diffraction textbook [1].

In our pole figure measurements, the sample was tilted from $\psi=0^{0}$ to $90^{\circ}$ with a step of $2^{\circ}$. At each $\psi$ position, the sample was rotated around its normal direction from $\phi=$ $0^{0}$ to $360^{\circ}$ at a step of $2^{\circ}$ while at the same time the XRD signal was collected by a detector at the fixed $2 \theta_{h k l}$ position. For instance, the $2 \theta_{110}$ angle is $38.55^{\circ}$ for detecting the $\mathrm{Nb}\{110\}$ plane. More experimental arrangement in this study was also described elsewhere [5,18]. Essentially, the XRD pole figure technique uses a "fixed length" of $\mathrm{x}$-ray scattering vector (by lock-in $2 \theta_{h k l}$ ) to map a family of $\{h k l\}$ points in reciprocal lattice space ( $k$ space).

Three samples were surveyed by a pole figure measurement. Figures $2(\mathrm{a}-\mathrm{c})$ are the experimental results of $\mathrm{Nb}\{110\}$ pole figures. Figure 2(a) is a pole figure of sample CED-034. It is the $\mathrm{Nb}$ (100) film on $\mathrm{MgO}$ (100) substrate. Figure 2(b) is sample Nb-SC-01. It is a single crystal bulk $\mathrm{Nb}$ (110) coupon. Figure 2(c) is sample Nb-PC-01. It is a polycrystalline bulk $\mathrm{Nb}$ coupon.

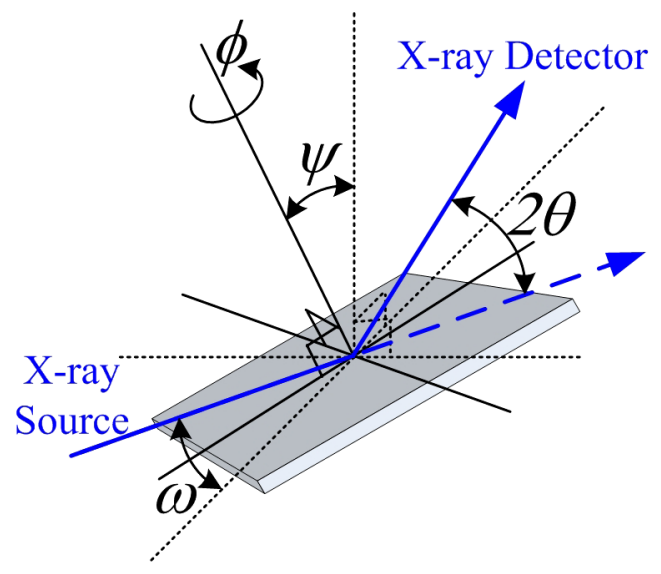

FIG. 1 (color online). Standard XRD experimental arrangement.
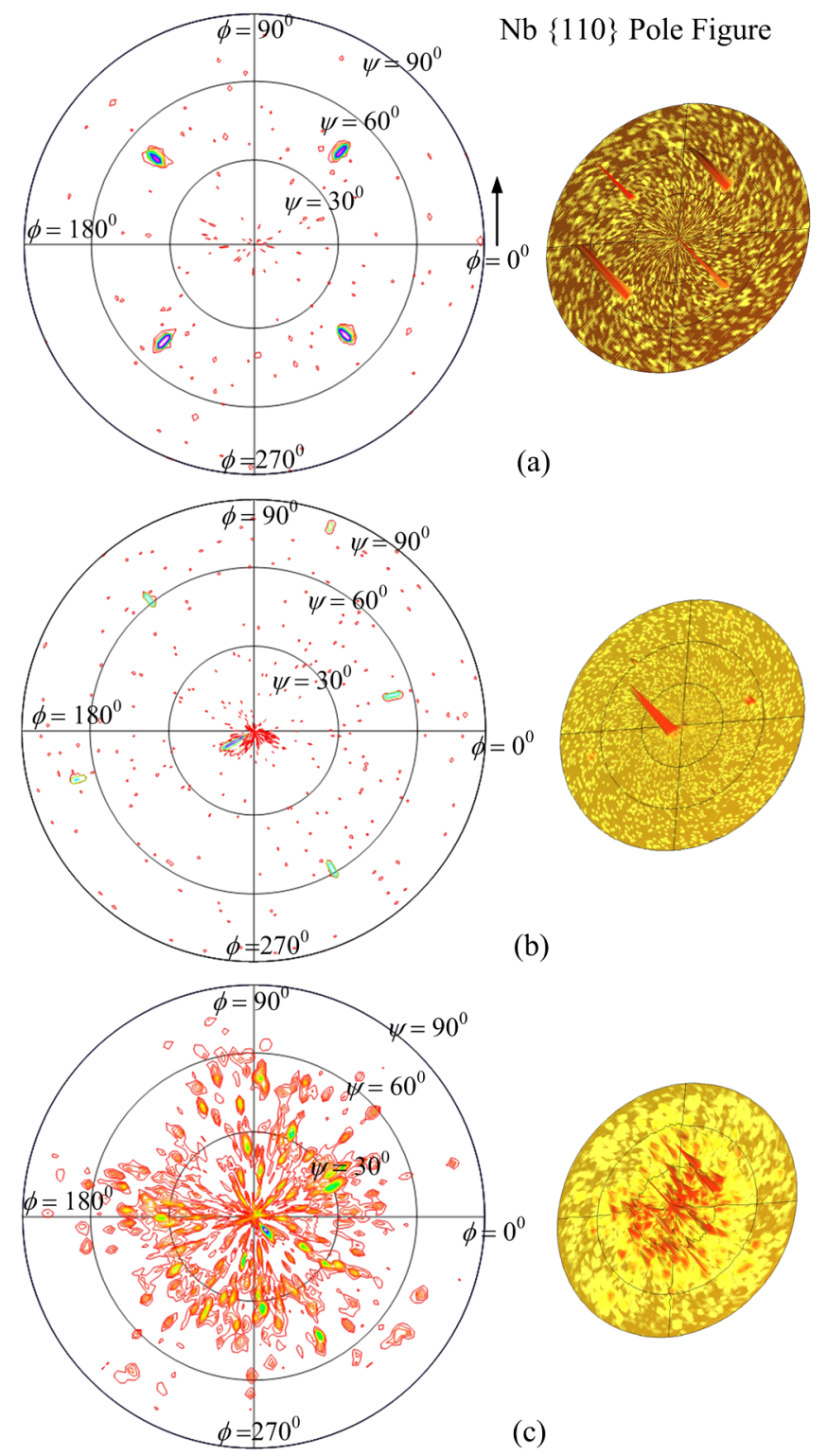

FIG. 2 (color online). Experimental $\mathrm{Nb}\{110\}$ pole figures. (a) Sample CED-034: a $\mathrm{Nb}(100)$ film on Mgo (100) substrate. (b) Sample Nb-SC-01: a single crystal bulk Nb (110) coupon. (c) Sample Nb-PC-01: a polycrystalline bulk Nb coupon. The right column shows a $3 \mathrm{D}$ view of the pole figures.

Figures 2(a) and 2(b) demonstrated that the samples are "single crystal." In the figures, a $\mathrm{Nb}(100)$ or a $\mathrm{Nb}(110)$ crystal plane is parallel to substrate cut surface (in plane), respectively. Figure 2(c) confirms that sample Nb-PC-01 is a polycrystalline material; the grains have no preferential orientation, or no obvious texture.

Reciprocal space mapping was used to characterize intragrain structures of three samples (film CED-34, bulk Nb-SC-01, and Nb-PC-01). A RSM plot usually shows a distribution of diffraction intensity of one $\{h k l\}$ point in reciprocal space. Structural information of the samples could be interpreted from a profile of the reciprocal lattice 
point. Experimental RSM data is presented in a twodimensional " $\omega \mathrm{vs} \omega / 2 \theta$ " plot. The $\omega / 2 \theta$ means the machine running under a coupled $\omega / 2 \theta$ scan mode, also known as a $\theta / 2 \theta$ (Bragg-Brentano) scan mode. Its $x$ coordinate represents variable $\theta$.

By Bragg's law, $2 d_{h k l \times} \sin \left(\theta_{h k l}\right)=\lambda_{\mathrm{Cu}, \mathrm{K} \alpha}, \Delta \theta_{h k l}$ is proportional to the distortion of lattice space $\left(\Delta d_{h k l}\right)$. A broadening effect along the $\theta_{h k l}$ coordinate indicates variation in $d_{h k l}$ (spacing of crystal planes $\{h k l\}$ ). $d_{h k l}$ is proportional to the lattice constant $(a)$ of $\mathrm{Nb}$, which is a body-centered cubic structure, and

$$
d_{h k l}=a / \sqrt{h^{2}+k^{2}+l^{2}}
$$

thus the $\mathrm{Nb}$ lattice distortion $\Delta a / a(\%)$ can be derived from $\Delta \theta_{h k l}$.

A broadening in the $\omega$ direction (or $\Delta \omega$ ) embodies a misorientation spread of a $\{h k l\}$ crystal plane, which implies structural imperfection in the sample (grain) due to "mosaic spread" (evidence of subgrains), dislocations population, bending, or polygonization of a lattice.

Before each RSM survey, an $\{h k l\}$ Bragg plane (or $\theta_{h k l}$ ), an X-ray incident angle $\left(\omega_{0}\right)$, an azimuth angle $(\phi)$, and a sample stage tilting angle $(\psi)$ were selected in order to probe a specific penetration depth in a particular sample. Then, the XRD machine scans in a $\omega / 2 \theta$ mode at different $\omega$ offset. Scan step of $\omega$ or $2 \theta$ is $0.06^{\circ}$.

To calculate penetration depth $(t)$ of an x-ray beam, this equation from Cullity's book [1] is applied:

$$
t=\sin \alpha / \mu \text {. }
$$

Here $\alpha$ is an incident angle, $\mu=1259$; it is the absorption coefficient of $\mathrm{Nb}$ at $\lambda_{\mathrm{Cu}, \mathrm{K} \alpha}=1.54 \AA$.

By controlling the penetration depth, we could either investigate the RSM of a surface layer, which is a few hundred nanometers, or probe that of a thicker (includes integration over the surface) layer. By comparison of RSM plots of a thinner layer to that of a thicker layer, one may discriminate the microstructure at different depths. This may reveal the structure evolution of the $\mathrm{Nb}$ sample after a coating or polishing.

For a shallow and a thick penetration survey, although different reciprocal points in $k$ space were observed via RSM, it is still reasonable and meaningful to compare profiles of the points. This is because (1) in the same sample measurement, the observed reciprocal points belong to the same $\{h k l\}$ crystal plane family; and (2) A Nb crystal lattice is a cubic structure, whose symmetry determines that a deviation of one $\{h k l\}$ shall lead to a proportional deviation of the other $\{h k l\}$ points, since the volume of a cubic "box" lattice shall be conservative.

It is also worth noting that such a comparison is only qualitative, in order to give a hint of crystallographic disorder. Because a strain of the lattice is always three dimensional, while the state-of-art RSM is a two-dimensional plot of such incoherence lattices, it is unrealistic to apply a 2D graph to depict a 3D lattice. This is not a problem particular to our RSM study but a conventional challenge to visualize 3D space structure by XRD.

The EBSD system being utilized in this study, being made by the EDAX/TSL company, is installed on an Amray scanning electron microscope at Jefferson Lab. The EBSD arrangement is equipped with EDAX/TSL company's OIM ${ }^{\mathrm{TM}}$ software to acquire and index the crystallographic orientations.

In this study, two parameters related to crystal quality were recorded for parallel comparison. The abbreviation of "Avg. CI" means the average confidence index; while "Avg. IQ" means the average image quality of the Kikuchi imaging pattern (an electron backscattering diffraction pattern). Higher CI means the TSL crystal orientation indexing software has a higher confidence to index the crystal zone and orientation, under that e-beam scan spot. Higher IQ means the Kikuchi imaging pattern (being originated at a single e-beam scan spot) is sharper. For a strained zone, then, a spot with a high density of crystallographic defects has a Kikuchi image quality worse than that of a perfect crystal zone.

\section{RESULTS AND DISCUSSION}

\section{A. Crystal quality evolution via film thickening \\ 1. Investigation of a $\mathrm{Nb}$ epitaxy film at different penetration depths by RSM}

In our previous studies [5,18], a trend became obvious that by coating a thicker $\mathrm{Nb}$ film, average crystal quality of a single crystal $\mathrm{Nb}$ film is advanced, as represented by the RRR value, a bulk electrical property.

It was suspected that the crystal quality of a film's top layer has been progressively improved, as the grains were being continuously thickened (in other words, the grain grows up). To confirm our speculation, we selected two

TABLE I. Two RSM experimental arrangements to measure $\{123\}$ reciprocal points at different x-ray penetration depths. Sample

\begin{tabular}{|c|c|c|c|c|c|c|c|}
\hline Arrangement & Omega $\omega\left(^{\circ}\right)$ & 2Theta, $2 \theta\left(^{\circ}\right)$ & Psi, $\psi\left(\left(^{\circ}\right)\right.$ & Phi, $\phi\left(^{\circ}\right)$ & $\begin{array}{c}\text { X-ray penetration } \\
\text { depth }\end{array}$ & $\begin{array}{l}\text { Spread of } \omega \text { at } \\
\text { FWHM, } \Delta \omega\left(^{\circ}\right)\end{array}$ & $\begin{array}{l}\text { Spread of } \theta \text { at } \\
\text { FWHM, } \Delta \theta\left(^{\circ}\right)\end{array}$ \\
\hline 1.1 & 3.1 & 121.82 & 0.61 & 28.45 & Shallow, $440 \mathrm{~nm}$ & 0.1 & 0.1 \\
\hline 1.2 & 27.3 & 121.87 & 58.32 & 27.65 & Thick, 33.65 um & 0.6 & 0.2 \\
\hline
\end{tabular}
label is CED-34. It is a $\mathrm{Nb}$ film on $\mathrm{MgO}(100)$ substrate. 
material analysis techniques (RSM and EBSD) to probe the crystal quality of sample $\mathrm{Nb}$ films.

Initially, $\mathrm{Nb}$ film sample CED-34 was surveyed by RSM. This film sample was coated by a typical Alameda Applied
Sciences Corporation CED deposition [5] method. Before coating, the magnesium oxide crystal substrate with $\mathrm{MgO}(100)$ in plane was annealed at $700^{\circ} \mathrm{C}$ for $12 \mathrm{~h}$. The substrate temperature was set at $500{ }^{\circ} \mathrm{C}$ during
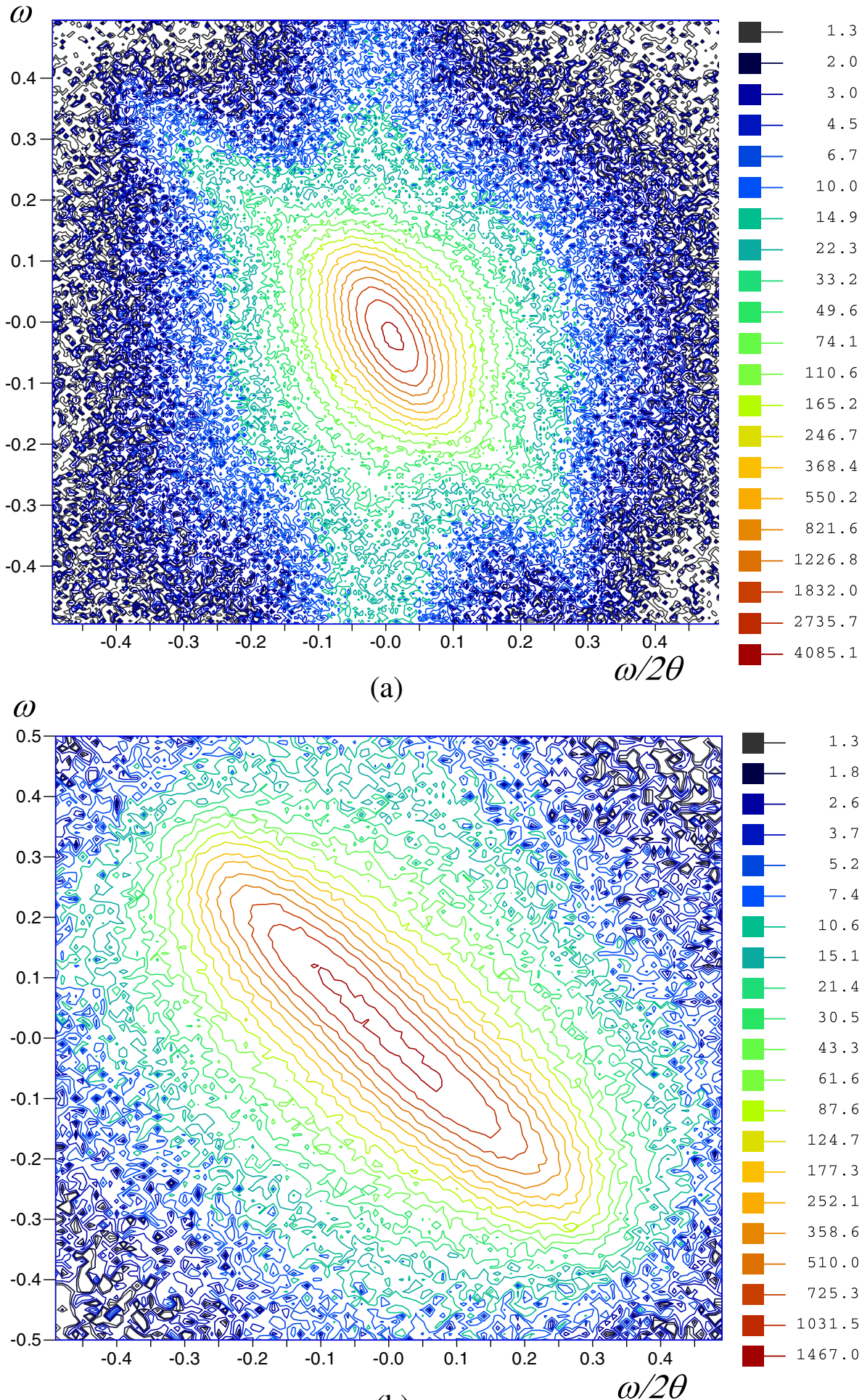

(b)

FIG. 3 (color online). RSM experimental plot of Nb film sample CED-034 which shows $\{123\}$ reciprocal points at different x-ray penetration depth: (a) shallow penetration; (b) deep penetration. 


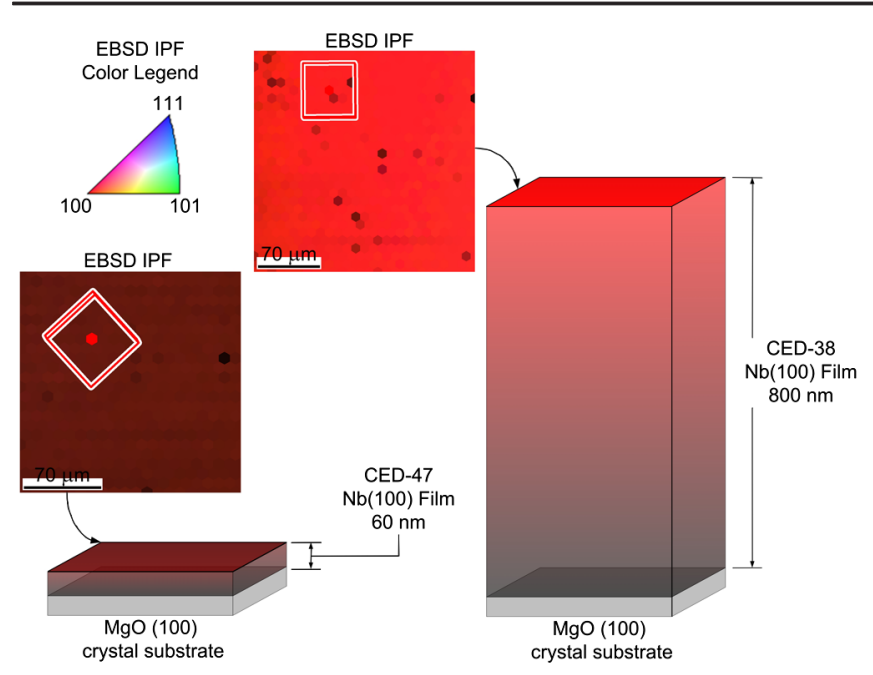

FIG. 4 (color online). Schematic illustration of $\mathrm{Nb}$ films CED-47 and CED-38. Their surface crystal quality was measured by EBSD. It indicates the $\mathrm{Nb}$ film crystal quality progressively evolves by thickening. They are the EBSD inversed pole figures of $\mathrm{Nb}$ film sample CED-47 and CED-38. EBSD survey area is $250 \times 250 \mu \mathrm{m}$, scan step $10 \mu \mathrm{m}$. IPF grayscales are rendered by IQ values; rendering scale is $[\min , \max ]=[500,2100]$.

deposition. The Nb film thickness was about $1.6 \mu \mathrm{m}$. The RRR value of the sample is 277 ; the superconducting transition temperature 9.21-9.25 K.

Through a pole figure measurement of $\mathrm{Nb}\{110\}$, and two $\phi$ scans of $\mathrm{Nb}\{110\}$ and $\mathrm{Mg} 110\}$ at orbital $\psi=60^{0}$, respectively, the epitaxial relationship between the $\mathrm{Nb}$ film and $\mathrm{MgO}$ substrate is revealed. It is $\mathrm{Nb}(001) / / \operatorname{MgO}(001)$ with $\mathrm{Nb}(110) / / \mathrm{Mg}(010)$, which

TABLE II. Crystal quality being measured by EBSD. It shows crystal quality progressively evolves by thickening a $\mathrm{Nb}$ film.

\begin{tabular}{lcc}
\hline \hline Sample label & CED-47 & CED-38 \\
\hline Thickness (nm) & $\sim 60$ & $\sim 800$ \\
RRR & 46 & 136 \\
$T_{c}(\mathrm{~K})$ & 8.95 & 9.20 \\
Average CI & 0.67 & 0.90 \\
Average IQ & 1121 & 2101 \\
Average misorientation angle & $0.18^{\circ}$ & $0.15^{\circ}$ \\
\hline \hline
\end{tabular}

was called the type $O_{p}$ epitaxial relationship in the Hutchinson's study $[19,20]$.

Table I shows two RSM experimental setups (1.1 and 1.2) on surveying sample CED-34. Both arrangements probed $\{123\}$ reciprocal points (by fixing $2 \theta_{123}=121.8^{\circ}$ ), but at different $k$ space positions. They have different probing depths.

For arrangement 1.1, the RSM survey probes a shallow surface layer (with penetration depth about $430 \mathrm{~nm}$ ). Figure 3 is the experimental RSM graph of sample CED-034 as described in Table I.

For the arrangement 1.2 (high incident angle $\omega$ ), theoretically the X-ray beam could probe a thicker layer $(\sim 3.6 \mu \mathrm{m}$ if all mass are $\mathrm{Nb}$ ). Because thickness of the $\mathrm{Nb}$ film CED-34 is only $1.6 \mu \mathrm{m}$, literally, the $\mathrm{x}$-ray beam had sampled the entire through-thickness of the film.

The deviation of lattice constant $(a)$ on the top surface versus that of the entire layer is

$$
\Delta a(\text { top-layer }) / \Delta a(\text { entire film }) \sim=0.1 / 0.2=1 / 2 .
$$

The crystal misorientation on the top surface versus that of the entire layer is

$$
\Delta \omega(\text { top-layer }) / \Delta \omega(\text { entire film }) \sim=0.1 / 0.6=1 / 6 .
$$

Figure 3(a) for arrangement 1.1 shows a reciprocal point $\{123\}$, whose profile is an oval near-circular shape. Sampling depth of arrangement 1.1 is shallow, about $430 \mathrm{~nm}$. The spread of $\omega$ and $2 \theta_{123}$ at FWHM, $\Delta \omega\left(^{\circ}\right)$ and $\Delta 2 \theta\left({ }^{\circ}\right)$, are both small $\left(\sim 0.1^{\circ}\right)$. The shape of arrangement 1.1 is similar to a singularity $k$-space point, which suggests that the surface structure of the film is close to a perfect crystal, if being compared to Fig. 2(b).

Figure 3(b) for set 1.2 shows a reciprocal pole $\{123\}$, whose profile is an asymmetrically elongated bar shape. Sampling depth of arrangement 1.2 is deep, the full $1.2 \mu \mathrm{m}$. The image obviously deviates from a $k$-space singularity point. It suggests that the entire film structure might be "bent" or gradually misoriented (spread of $\omega$ at FWHM, $\Delta \omega=\sim 0.6^{\circ}$ ).

By comparing the RSM figure of 3(a) to 3(b), it demonstrated that the crystal structure of the top layer of $\mathrm{Nb}$ film CED-34 is superior to that of the average ones, regarding the entire film.

TABLE III. Two RSM experimental arrangements to measure $\{110\}$ reciprocal points at different x-ray penetration depths. Sample label is $\mathrm{Nb}-\mathrm{SC}-01$. It is a single crystal bulk $\mathrm{Nb}(100)$ coupon sample.

\begin{tabular}{lccccccc}
\hline \hline Arrangement & Omega $\omega\left(^{\circ}\right)$ & 2Theta, $2 \theta_{110}\left(^{\circ}\right)$ & Psi, $\psi\left(^{\circ}\right)$ & Phi, $\phi\left(^{\circ}\right)$ & $\begin{array}{c}\text { X-ray penetration } \\
\text { depth }\end{array}$ & $\begin{array}{c}\text { Spread of } \omega \\
\text { at FWHM, } \Delta \omega\left(^{\circ}\right)\end{array}$ & $\begin{array}{c}\text { Spread of } \theta \\
\text { at FWHM, } \Delta \theta\left(^{\circ}\right)\end{array}$ \\
\hline 2.2 & 19.28 & 38.551 & 83.2 & 69.34 & Shallow, $\sim 310 \mathrm{~nm}$ & $0.6^{b}$ & 0.2 \\
2.1 & 20.53 & 38.551 & $-8.12^{a}$ & 0 & Thick, $\sim 2.75 \mu \mathrm{m}$ & 0.1 & 0.1 \\
\hline
\end{tabular}

${ }^{\mathrm{a}}$ Minus sign means tilting direction.

${ }^{\mathrm{b}}$ Spread of multiple subpeaks. 


\section{Investigation of two $\mathrm{Nb}$ epitaxy films of different thickness by EBSD}

To verify whether crystal quality does advance through a film thickening process, the EBSD technique was also applied to measure the crystal quality of two films of different deposition thickness.

The films (CED-47 and CED-38) were created by the same CED deposition conditions as that of CED-34,
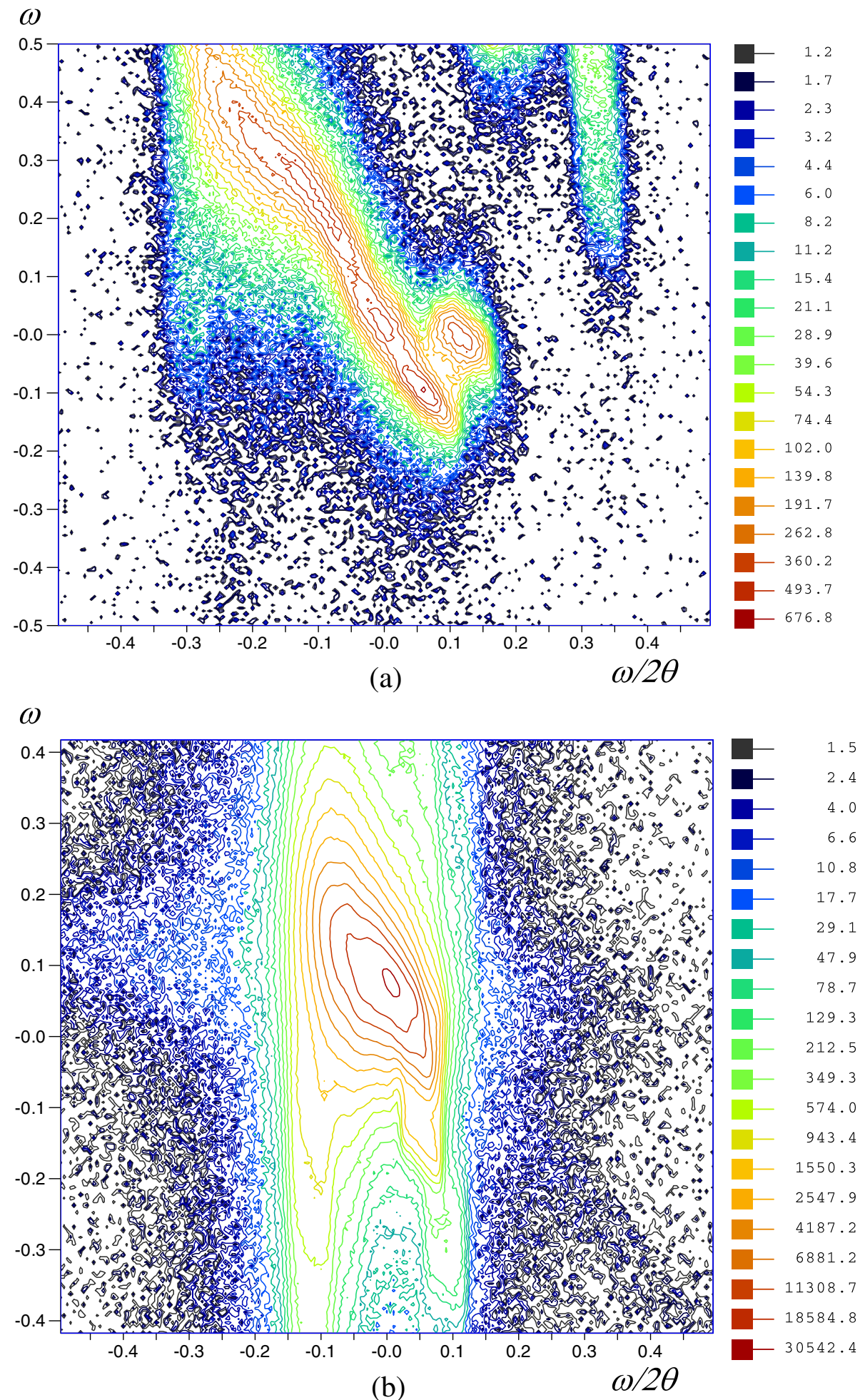

FIG. 5 (color online). Experimental RSM graph of bulk sample Nb-SC-01 as described in Table III. (a) Shallow penetration; (b) deep penetration. 
aforementioned in the Experimental Section and Sec. 3.1.1, except their thicknesses are 60 and $800 \mathrm{~nm}$, respectively.

Figure 4 shows the samples' EBSD inversed pole figures (IPF). The figures also illustrate thickness difference of the samples. The EBSD survey area is $250 \times 250 \mu \mathrm{m}$, scan step $10 \mu \mathrm{m}$. The IPF graphs' grayscale are rendered by IQ values, whose rendering scale is $[\min , \max ]=$ $[500,2100]$. Bright red color means the scanning zone has a higher crystal quality. Dark red zones are low crystal quality ones.

Table II lists some measurable parameters given by TSL OIM software (being extracted out of the raw data of Fig. 4). The table shows the RRR values, an averaged figure of merit to evaluate electrical conductivity, or the electronmean free path in the state of normal conductivity, increased up from 46 to 136 ( $\sim 3$ times). The superconducting transition temperature $\left(T_{c}\right)$ of the $\mathrm{Nb}$ films increased from 8.95 to $9.2 \mathrm{~K}$, which also suggested the average crystallographic defect density of $\mathrm{Nb}$ film being suppressed via a film thickening.

The table indicates the average confidence index changes from 0.67 to 0.9 ; while the average image quality has a more obvious change, which is from 1121 to 2101 (about 2 times increment). The average misorientation angle of the scanning area $(250 \times 250 \mu \mathrm{m})$, is smaller than that of the thicker film. But such change (0.18 vs 0.15$)$ is not obvious. It shows the EBSD technique is not efficient at revealing small misorientation.

Since the probing thickness of EBSD is only about $50 \mathrm{~nm}$, and all the EBSD parameters relevant to crystal quality indicated a trend of progression, it substantiated that crystal quality gradually improved as the film thickening progressed. Both RSM and EBSD experiments demonstrated the top layers crystal quality has less crystallographic defect density than that on the interface of the $\mathrm{Nb}$ film or $\mathrm{MgO}$ substrate.

\section{B. Investigation of a single crystal bulk $\mathrm{Nb}$ coupon sample at different penetration depths by RSM}

Table III shows two RSM experimental setups (2.1 and 2.2), which have different probing depths on bulk sample $\mathrm{Nb}-\mathrm{SC}-01$. Both arrangements were probing $\{110\}$ reciprocal poles (by fixing $2 \theta_{110}=38.5^{\circ}$ ), at different $k$-space points.

Figure 5 shows the experimental RSM graphs of bulk sample Nb-SC-01. For arrangement 2.1, it probes a shallow surface (with penetration depth about $310 \mathrm{~nm}$. Figure 5(a) shows multiple peaks at a reciprocal point $\{110\}$, whose profile is an asymmetrical, widespread, and multicentered contour. This RSM plot is far from that of perfect crystal. Such a multiple-peak image might be explained by formation of subgrains on the surface, with each one having a slightly different orientation (misorientation angles $\sim 0.1^{\circ}$ ). As is known in metallurgy, a subgrain is a portion of a crystal or grain slightly different in orientation from neighboring portions of the same crystal. Usually, neighboring subgrains are separated by low-angle boundaries.

The image's obvious deviation from a $k$-space singularity point suggests that the adopted CMP process plus a light BCP process still produced a damaged surface structure (strain or subgrains). The subgrains may be gradually misoriented (spread of $\omega$ at FWHM, $\Delta \omega\left(^{\circ}\right)=\sim 0.6^{\circ}$ ).

The setup 2.2 (high incident angle) probes a thicker layer (2.76 $\mu \mathrm{m}$ if all mass is $\mathrm{Nb}$ ). Figure 5(b) shows one peak of reciprocal pole $\{110\}$, whose profile is in quasicircular shape. Its max peak is similar to a singularity $k$-space point.

The spread of $\omega$ and $2 \theta_{110}$ at FWHM, $\Delta \omega\left(^{\circ}\right)$ and $\Delta 2 \theta\left(^{\circ}\right)$, are both small $\left(\sim 0.1^{\circ}\right)$. Its shape is similar to a singularity $k$-space point, which suggests that bulk structure is close to a perfect crystal, if being compared to Fig. 5(a).

The deviation of lattice constant $(a)$ on a top surface versus that of an entire layer is

$$
\Delta a(\text { top-layer }) / \Delta a(\text { entire film }) \sim=0.2 / 0.1=2 / 1
$$

The crystal misorientation on the top surface layer versus that of entire layer is

$$
\Delta \omega \text { (top-layer }) / \Delta \omega(\text { entire film }) \sim=0.6 / 0.1=6 / 1 .
$$

Thus, the RSM Figs. 5(a) vs 5(b) demonstrated that the top layer's crystal structure of the mirror-finished bulk $\mathrm{Nb}$ is crystallographically disordered, and the top layer crystal structure is inferior to that of the deep layer, regarding the intragrain crystal quality.

\section{Investigation of a polycrystalline bulk Nb coupon sample at different penetration depth by RSM}

Table IV shows two RSM experimental setups (3.1 and 3.2), which have different probing depth on bulk sample Nb-PC-01. Both arrangements were probing $\{110\}$ reciprocal points (by fixing $2 \theta_{110}=38.5^{\circ}$ ), but at different $k$-space points. Because it is a polycrystalline (with polygrains) material, it does not matter how to set $\psi$ or $\varphi$ angles.

TABLE IV. Two RSM experimental arrangements to measure $\{110\}$ reciprocal points at different x-ray penetration depth. Sample label is $\mathrm{Nb}-\mathrm{PC}-01$. It is a polycrystalline bulk $\mathrm{Nb}$ sample.

\begin{tabular}{lccccc}
\hline \hline Arrangement & Omega $\omega\left(^{\circ}\right)$ & 2Theta, $2 \theta_{110}\left({ }^{\circ}\right)$ & Psi, $\psi\left({ }^{\circ}\right)$ & Phi, $\phi\left(^{\circ}\right)$ & X-ray penetration depth \\
\hline 3.1 & 1.5000 & 38.551 & 0 & 0 & Shallow, $208 \mathrm{~nm}$ \\
3.2 & 19.2755 & 38.551 & 0 & 0 & Thick, $2.62 \mu \mathrm{m}$ \\
\hline \hline
\end{tabular}



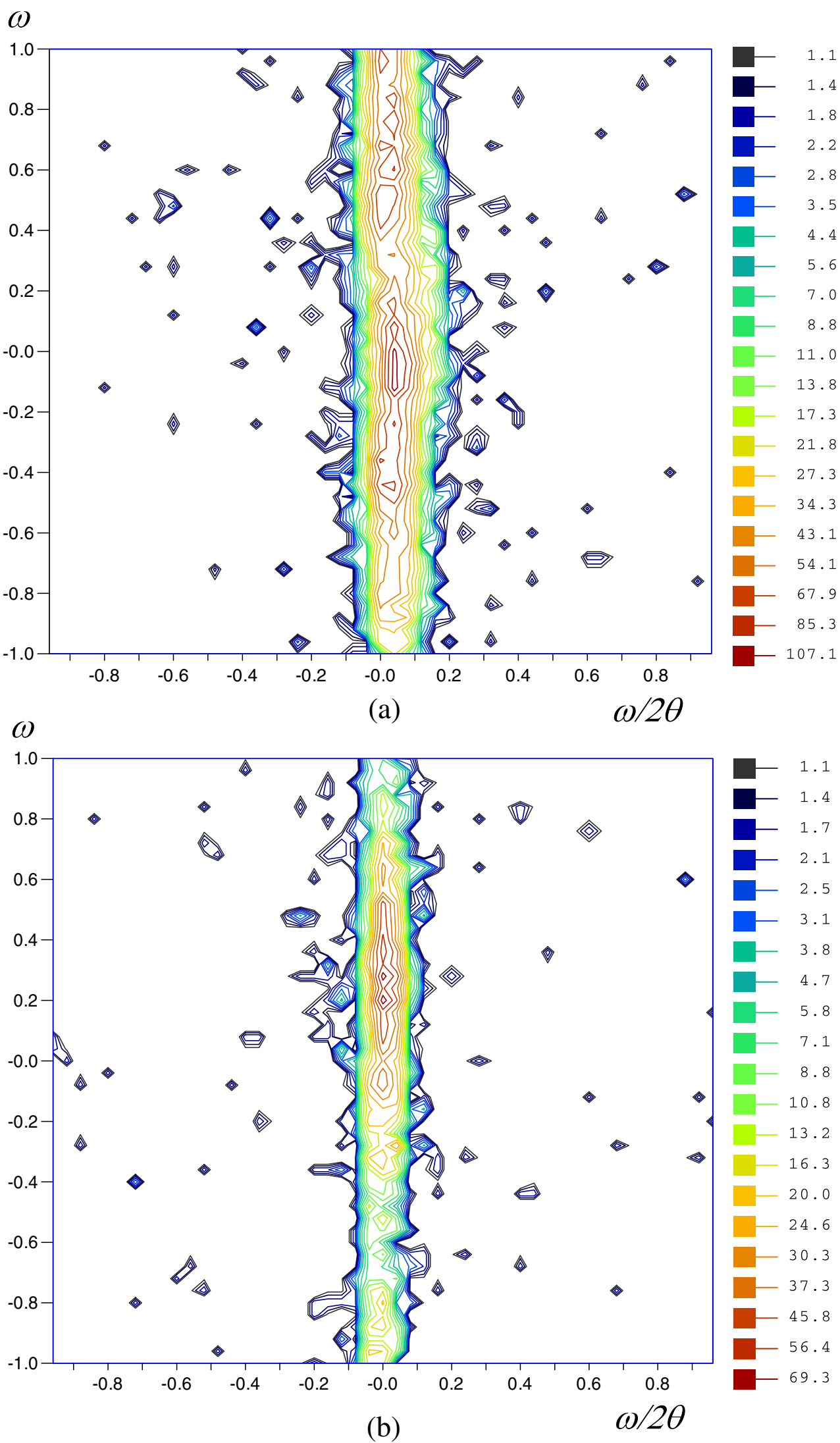

FIG. 6 (color online). Experimental RSM graphs of bulk polycrystalline sample Nb-PC-01 as described in Table IV. (a) Shallow penetration; (b) deep penetration. 
For arrangement 3.1, the x-ray beam probes a shallow surface (with penetration depth about $208 \mathrm{~nm}$ ). The x-ray beam sampled the top surface damage layer. Figure 6 presents the experimental RSM graphs as described in Table IV. For the setup 3.2 (high incident angle), the x-ray beam probes a thicker layer $(2.62 \mu \mathrm{m})$.

Figures 6(a) and 6(b) show similar "stripes" in $k$ space, which is a typical sign of polygrain materials, for the grains are misoriented gradually along the $\omega$ coordinate. The appearance of (a) and (b) are similar, although the stripe (a) is quite significantly broader in the $\omega$ direction, and wider in the spread of $\theta_{110}$ (in the $\omega / 2 \theta$ coordinate) than that of (b). As mentioned previously, a broadening effect of $\theta_{110}$ implies a larger lattice constant deviation. Intuitively, the plot (a-b) implies that the average crystal quality of deep-layer $\mathrm{Nb}$ is better than that of the surface layer, which might still have a damaged area even after being polished. It is hard to describe the difference quantitatively. Nevertheless, such observation of the spread of the stripe is consistent with residual surface damage.

Also note, the averaged FWHM width of $\theta_{110}$ (in the $\omega / 2 \theta$ coordinate) of Fig. 6(b) is similar to that of Fig. 5(b). This suggests that in a deep layer far from the surface the average crystal quality of polycrystalline $\mathrm{Nb}-\mathrm{PC}-01$ is comparable to that of the single grain $\mathrm{Nb}-\mathrm{SC}-01$.

\section{CONCLUSION}

We have shown that an XRD RSM measurement is a useful nondestructive technique to measure intragrain crystal quality of $\mathrm{Nb}$ surfaces. By using different $\mathrm{x}$-ray penetration depths, crystal quality at different thickness can be discerned. This technique is efficient and useful to probe SRF materials such as $\mathrm{Nb}$ films or bulk single crystal small samples.

This study confirmed that during a $\mathrm{Nb}$ film coating process, the film thickening process, via energetic deposition, can advance the crystal quality of a film's topmost surface layer, which is of greatest interest for many applications.

For an ultrathin film (say $\ll 50 \mathrm{~nm}$ ), the microstructural disorder is largely influenced by the interface; by depositing a much thicker film (say $>1 \mu \mathrm{m}$ ), the topmost layer of $50 \mathrm{~nm}$ may obtain a well-ordered structure. Such a phenomenon suggests a micron thick film coating shall be suitable for SRF cavities.

The RSM study also confirmed that a mechanical mirror polishing introduces substantial microstructure disorders into the bulk Nb material's topmost layer. Chemical polishing or etching is a way to remove the surface damage layer. The particular CMP process plus BCP process used on samples here still left a slightly damaged surface. Process development feedback from the RSM technique described here may guide optimization of optimal surface processing of bulk materials in addition to thin film materials. A very recent study on high temperature treatment of a large grain SRF $\mathrm{Nb}$ cavity has successfully adopted the RSM and pole figure techniques to reveal the surface structure-properties relationship [21].

\section{ACKNOWLEDGMENTS}

This research is supported at AASC by DOE via Grants No. DE-FG02-08ER85162 and No. DE-SC0004994. The JLab effort was provided by Jefferson Science Associates, LLC, under U.S. DOE Contract No. DEAC05-06OR23177, including supplemental funding provided by the American Recovery and Reinvestment Act.

[1] B. D. Cullity and S. R. Stock, Elements of X-Ray Diffraction (Prentice Hall, New Jersey, 2001), p. 347.

[2] E. H. Snell, D. B. Henry, and E. O. B. Gloria, and H. Snell, Methods Enzymol. 368, 268 (2003).

[3] L. P. X. Zhao, C. E. Reece, K. Seo, M. Krishnan, and E. Valderrama, J. Appl. Phys. 112, 016102 (2012).

[4] X. Zhao, L. Phillips, C. E. Reece, K. Seo, M. Krishnan, and E. Valderrama, Jpn. J. Appl. Phys. 110, 033523 (2011).

[5] M. Krishnan, E. Valderrama, B. Bures, K. Wilson-Elliott, X. Zhao, L. Phillips, A.-M. Valente-Feliciano, J. Spradlin, C. Reece, and K. Seo, Supercond. Sci. Technol. 24, 115002 (2011).

[6] X. Zhao, A.-M. Valente-Feliciano, C. Xu, R. L. Geng, L. Phillips, C. E. Reece, K. Seo, R. Crooks, M. Krishnan, A. Gerhan, B. Bures, K. W. Elliott, and J. Wright, J. Vac. Sci. Technol. A27 620 (2009).

[7] M. Krishnan, B. Bures, K. Wilson Elliott, S. Leandro, X. Zhao, A.-M. Valente-Feliciano, L. Phillips, B. Xiao, C. Reece, and K. Seo, in Proceedings of the 14th International Conference on RF Superconductivity (SRF-2009) Berlin, Germany, 2009 (unpublished).

[8] G. Wu, L. Phillips, R. Sundelin, and A.-M. Valente, in Proceedings of the 2003 Particle Accelerator Conference, Portland, OR (IEEE, New York, 2003).

[9] V. Randle, in Electron Backscatter Diffraction in Materials Science, edited by A. J. Schwartz, M. Kumar, and B. L. Adams (Springer, New York, 2000).

[10] X. Zhao, G. Ciovati, and T. R. Bieler, Phys. Rev. ST Accel. Beams 13, 124701 (2010).

[11] T. Bieler, Phys. Rev. ST Accel. Beams 13, 031002 (2010).

[12] H. Yoshida, K. Inaba, and N. Sato, Appl. Phys. Lett. 90, 181930 (2007).

[13] A. Tersigni, X. R. Qin, C.-Y. Kim, R. A. Gordon, and D. T. Jiang, Phys. Rev. B 84, 035303 (2011).

[14] J. F. W. a. A. Kharchenko (unpublished).

[15] D.-M. Smilgies, D. R. Blasini, S. Hottac, and H. Yanagi, J. Synchrotron Radiat. 12, 807 (2005).

[16] P. F. Fewster, in Newsletter No. 24, December 2000, Commission on Powder Diffraction, International Union of Crystallography (2000).

[17] E. Koppensteiner, G. Bauer, H. Kibbel, and e. Kasper, J. Appl. Phys. 76, 3489 (1994). 
[18] M. Krishnan, E. Valderrama, C. James, X. Zhao, J. Spradlin, A.-M. Valente Feliciano, L. Phillips, C. E. Reece, K. Seo, and Z. H. Sung, Phys. Rev. ST Accel. Beams 15, 032001 (2012).

[19] T. E. Hutchinson and K. H. Olsen, J. Appl. Phys. 38, 4933 (1967).
[20] Hutchinson, J. Appl. Phys. 36, 270 (1965).

[21] G. C. P. Dhakal, G. R. Myneni, K. E. Gray, N. Groll, P. Maheshwari, D. M. McRae, R. Pike, T. Proslier, F. Stevie, R. P. Walsh, Q. Yang, and J. Zasadzinzki, Phys. Rev. ST Accel. Beams 16, 13 (2013). 